\title{
Electronic structure and identification of deep defects in GaP
}

\author{
M. Scheffler, ${ }^{*}$ J. Bernholc, ${ }^{\dagger}$ N. O. Lipari, and S. T. Pantelides \\ IBM Thomas J. Watson Research Center, Yorktown Heights, New York, 10598 \\ (Received 25 February 1983; revised manuscript received 14 October 1983)
}

\begin{abstract}
We report a theoretical study of the Ga vacancy, the $\mathbf{P}$ antisite defect (i.e., a $\mathbf{P}$ atom occupying a normally $\mathrm{Ga}$ site), and carbon impurities at $\mathrm{Ga}$ sites. The analysis employs a modified version of the self-consistent Green's-function method that was previously used to describe deep centers in Si. In all three cases, the number, orbital content, and relative energy positions of the localized states can be understood in terms of simple models, as in the case of corresponding centers in Si. In particular, we find that, in all cases, the gap state consists primarily of atomiclike $p$ orbitals centered on the four nearest neighbors, revealing that deviations from $s p^{3}$-hybrid character are possible even without any relaxation of the surrounding lattice. In the case of the antisite defect, we find an $A_{1}$ bound state in the gap which is consistent with the EPR spectra that led to the identification of the defect. The calculated ionization energies are in agreement with optical data and more recent deeplevel-transient-spectroscopy data. In the case of the $\mathrm{Ga}$ vacancy, we find a $T_{2}$ level in the gap above an $A_{1}$ resonance inside the valence bands, and in the case of carbon, we find a deep $A_{1}$ level in the gap. These results contradict key assumptions that had led to the identification of the NRL-1 EPR signal as the $V^{2-}$ charge state of the Ga vacancy. More recent experiments redetermined some of the parameters of NRL-1 and reassigned the center to the neutral state of the Ga vacancy. Here, we discuss the interplay between experiment and theory that led to the new identification and describe existing difficulties that limit our ability to construct a detailed model which is consistent with all experiments and with theory.
\end{abstract}

\section{INTRODUCTION}

Studies of deep impurities and defects in compound semiconductors have long been hampered by experimental difficulties in establishing their identity and by the general absence of adequate theoretical treatments. A major step toward the positive identification of defects in III-V compounds was accomplished recently when electronparamagnetic-resonance (EPR) experiments became possible in these materials. ${ }^{1-3}$ These experiments led to the identification of the $\mathbf{P}$ antisite defect in GaP (Refs. 1 and 2) (i.e., a $P$ atom occupying a normally $\mathrm{Ga}$ site), the $\mathrm{Ga}$ vacancy, ${ }^{3}$ and, more recently, the As antisite defect in GaAs. ${ }^{4,5}$ Theoretical developments also have contributed to the process of identifying and understanding the properties of several defects. Van Vechten ${ }^{6}$ developed models of the thermochemistry of defect formation and was able to predict which defects are most likely to form under different crystal-growth conditions. His prediction that antisite-related defects are very likely to form and act as efficient recombination centers in fact precipitated the later identification of such defects. ${ }^{1,2}$

During the last few years, theoretical self-consistent Green's-function methods were developed ${ }^{7,8}$ which provided accurate descriptions of the electronic structure of deep defects and impurities. ${ }^{9}$ Initially, these methods were applied to the vacancy and deep impurities in $\mathrm{Si}^{7-10}$ More recently, we modified the method of Refs. 7 and 10 and used it for a series of studies of defects and impurities in GaP. ${ }^{11}$ These studies demonstrated that accurate calculations can be a valuable tool in the process of defect identification.
Our theoretical calculations concentrated first on the $\mathbf{P}$ antisite defect and the $\mathrm{Ga}$ vacancy, both of which had been identified from analysis of EPR spectra. ${ }^{1-3}$ Our results confirmed the identification of the antisite defect, but challenged the assignment of the EPR spectrum labeled NRL-1 to the doubly-negatively-charged state of the Ga vacancy $\left(V^{2-}\right)$ because the experimental charcteristics of NRL-1 were inconsistent with the theoretical properties of the vacancy. In particular, NRL-1 was found to originate from a center with full tetrahedral symmetry and electronic spin $\frac{1}{2}$, whereas theory determined that all spin- $\frac{1}{2}$ charge states of the vacancy are either unstable against Jahn-Teller distortions or are ruled out by other experimental data. We then carried out calculations for carbon impurities at $\mathrm{Ga}$ sites $\left(C_{\mathrm{Ga}}\right)$ and found that all their properties were consistent with those of NRL-1. Since we first reported this work, ${ }^{11}$ however, new measurements have resulted in substantial changes in the properties attributed to NRL-1. Most significantly, lowtemperature uniaxial-stress measurements ${ }^{12}$ have determined that the electronic spin of the center responsible for NRL-1 is actually $\frac{3}{2}$. This change rules out both $V^{2-}$ and $\mathrm{C}_{\mathrm{Ga}}$ and led to the suggestion ${ }^{12}$ that the EPR-active center is the neutral Ga vacancy which has a spin- $\frac{3}{2}$ state that is stable against Jahn-Teller distortions. We subsequently pointed out to the authors of Ref. 12 that this identification still remains inconsistent with the published properties of NRL- 1 in $n$-type samples. We have since been informed ${ }^{13}$ that reexamination of these data has revealed that the properties of NRL- 1 in $n$-type samples are not as reported in Ref. 3. Finally, the capture cross section of a center detected by deep-level transient spectroscopy 
(DLTS) and tentatively identified as the source of NRL-1 (Ref. 14) was recently determined ${ }^{15}$ to be 2 orders of magnitude smaller than what had been reported earlier. ${ }^{14} \mathrm{~A}$ review of all the currently available experimental information reveals that the neutral Ga vacancy is the most likely center that gives rise to the NRL-1 EPR signal.

The plan of this paper is as follows. In Sec. II we describe the Green's-function method and the modifications of the basic technique ${ }^{7,10}$ that were introduced for the calculations of the Ga vacancy, the $P$ antisite defect, and carbon substitutional impurities at $\mathrm{Ga}$ sites. In Sec. III we give a detailed description of the electronic structure of these three defects and explain the localized states of each defect in terms of a simple physical model. In the case of the antisite defect, we confirm its identification and find that the theoretical ionization energies are in excellent agreement with optical and more recent DLTS data. In the cases of the Ga vacancy and carbon we discuss in detail the developments surrounding the identification of the EPR signal labeled NRL-1 and describe the role of the theoretical results in the identification process.

\section{GREEN'S-FUNCTION METHOD AND CALCULATIONS}

The Green's-function method used for the present investigation is a modified version of the one used previously to study defects and impurities in $\mathrm{Si}^{7,10}$ The two versions are based on the same formalism, ${ }^{16}$ but differ in the choice of basis sets used to represent the operators in matrix form for the actual calculations. Here, we summarize the main equations of the formalism for completeness.

\section{A. Green's-function formalism}

Let $\mathscr{H}$ be the Hamiltonian operator describing a given multiatomic system in the one-electron approximation. The corresponding Green's operator is defined by

$$
\mathscr{G}(E)=\lim _{\epsilon \rightarrow 0^{+}}(E+i \epsilon-\mathscr{P})^{-1},
$$

where the procedure of taking the limit as $\epsilon \rightarrow 0^{+}$is necessary only when $E$ is an eigenvalue of $\mathscr{H}$, i.e., where $(E-\mathscr{H})^{-1}$ is singular. It follows that if the system has any discrete eigenvalues, they are given by the poles of $\mathscr{G}(E)$, i.e., by the zeros of the determinant of $\mathscr{G}^{-1}(E)$. If the system has a continuum of states, the charge-density operator is given by

$$
\rho(E)=-\frac{2}{\pi} \operatorname{Im} \mathscr{G}(E),
$$

so that the state density is

$$
N(E)=\operatorname{Tr} \rho(E),
$$

or

$$
N(E)=-\frac{2}{\pi} \operatorname{Tr} \operatorname{Im} \mathscr{G}(E) .
$$

These equations can be specialized in the case of a perfect crystal whose one-electron Hamiltonian is $H^{0}$ and the same crystal containing a defect, in which case the Hamiltonian can be written as

$$
H=H+U \text {. }
$$

The corresponding Green's operators $G^{0}$ and $G$, defined by (1) in terms of $H^{0}$ and $H$, respectively, are then related by Dyson's equation

$$
G(E)=G^{0}(E)+G^{0}(E) U G(E) .
$$

This equation can be solved formally to yield

$$
G(E)=\left[1-G^{0}(E) U\right]^{-1} G^{0}(E) .
$$

It follows that the crystal containing a defect has the same band continua as the host crystal. Furthermore, in the energy gaps there will be defect-induced discrete states at energies where $\left(1-G^{0} U\right)^{-1}$ is singular, i.e., where the determinant

$$
D(E)=\operatorname{det}|| 1-G^{0}(E) U||
$$

is equal to zero. For each state, the wave function is given by

$$
\left[1-G^{0}(E) U\right] \psi=0 .
$$

Within the band continua, the defect-induced change in the density of states is given by

$$
\begin{aligned}
\Delta N(E) & =-\frac{2}{\pi} \operatorname{Tr} \operatorname{Im}\left[G(E)-G^{0}(E)\right] \\
& =-\frac{2}{\pi} \operatorname{Tr} \operatorname{Im}\left\{G^{0}(E) U\left[1-G^{0}(E) U\right]^{-1} G^{0}(E)\right\} .
\end{aligned}
$$

One can show ${ }^{16}$ that $\Delta N(E)$ can also be written as

$$
\Delta N(E)=\frac{2}{\pi} \frac{d \delta(E)}{d E},
$$

where the phase shift $\delta(E)$ is defined by

$$
\delta(E)=-\tan ^{-1}[\operatorname{Im} D(E) / \operatorname{Re} D(E)] .
$$

Finally, the change in the charge density arising from redistributions in the valence bands is given by the operator

$\Delta \rho_{v}=-\frac{2}{\pi} \operatorname{Im} \int_{-\infty}^{E_{v}} G^{0}(E) U\left[1-G^{0}(E) U\right]^{-1} G^{0}(E)$,

where $E_{v}$ is the top of the valence bands. The total change in the charge density is given by the operator

$$
\Delta \rho=\Delta \rho_{v}+\sum_{i}\left|\psi_{i}\right\rangle\left\langle\psi_{i}\right|,
$$

where $\psi_{i}$ are the wave functions of the occupied discrete states in the energy gaps.

In order to carry out calculations with the above formalism, the operators $G^{0}(E)$ and $U$ must be represented in matrix form. For this purpose, it is convenient to write $G^{0}(E)$ in the so-called spectral representation, namely,

$$
G^{0}(E)=\lim _{\epsilon \rightarrow 0^{+}} \sum_{n, \overrightarrow{\mathrm{k}}} \frac{|n, \overrightarrow{\mathrm{k}}\rangle\langle n, \overrightarrow{\mathrm{k}}|}{E+i \epsilon-E_{n, \overrightarrow{\mathrm{k}}}},
$$

where $E_{n, \overrightarrow{\mathrm{k}}}$ and $|n, \overrightarrow{\mathrm{k}}\rangle$ are the energy bands and Bloch functions, respectively. Using the Dirac identity to take the limit as $\epsilon \rightarrow 0^{+}$, one obtains 


$$
G^{0}(E)=\mathrm{P} \int \frac{A\left(E^{\prime}\right)}{E-E^{\prime}} d E^{\prime}-i \pi A(E)
$$

where $\mathbf{P}$ stands for principal part of the singular integral, and $A(E)$, the spectral-density operator, is given by

$$
A(E)=\sum_{n, \overrightarrow{\mathrm{k}}}|n, \overrightarrow{\mathrm{k}}\rangle\langle n, \overrightarrow{\mathrm{k}}| \delta\left(E-E_{n, \overrightarrow{\mathrm{k}}}\right) .
$$

The calculations can then be performed by choosing a basis set of functions $\left\{\phi_{\alpha}\right\}$ and evaluating the matrices $G_{\alpha \alpha^{\prime}}^{0}(E)$ and $U_{\alpha \alpha^{\prime}}$. For self-consistent calculations, the calculation is first done with a trial $U$. The induced change in the charge density is then calculated from Eq. (14). This charge density is used to obtain a new $U$ in the local-density approximation ${ }^{17,18}$ and the process is repeated until self-consistency is achieved. The power and efficiency of the method lie in the fact that, for localized potentials $U, U_{\alpha \alpha^{\prime}}$ is nonzero only for a small number of basis functions, and that one need keep $G_{\alpha \alpha^{\prime}}^{0}(E)$ only in the subspace of these functions. ${ }^{7,8,14}$

\section{B. Choice of basis sets}

As we saw in the preceding subsection, in order to calculate the Green's function of the host crystal, one must first carry out an energy-band calculation to determine the energies $E_{n, \overrightarrow{\mathrm{k}}}$ and the Bloch functions $|n, \overrightarrow{\mathrm{k}}\rangle$. Such a calculation is usually performed by expanding the Bloch functions in terms of a basis set which is appropriate for the perfect crystal in the energy region of interest. Once this calculation is done, one must then choose the set $\left\{\phi_{\alpha}\right\}$ to represent $G^{0}(E)$ and $U$ in matrix form in order to carry out the defect calculation. In the original applications of the method to defects and impurities in $\mathrm{Si}$ by Bernholc et al., ${ }^{7,10}$ a set of localized orbtials centered on each atom was chosen for the energy-band calculation, and then the same set of orbitals was used for the defect calculations. Because of the localization of $U$, only orbitals centered on the first few shells of neighbors needed to be kept. ${ }^{7,10}$ In the present work we removed the restriction that the same basis set be used for both the energy-band calculation and the defect calculation. Thus we carry out the energy-band calculation using a plane-wave basis set, which is particularly convenient for systematic convergence tests. Once the energy-band calculation has been performed, the defect calculations are performed by choosing a set of localized functions $\left\{\phi_{\alpha}\right\}$ to represent $G^{0}$ and $U$ in matrix form.

\section{Calculations}

The one-electron Hamiltonian for $\mathrm{GaP}$ is written in the form

$$
\begin{aligned}
H^{0}= & -\nabla^{2}+\sum_{j}\left[v_{\mathrm{Ga}}^{\mathrm{ion}}\left(\overrightarrow{\mathrm{r}}-\overrightarrow{\mathbf{R}}_{j}\right)+v_{\mathrm{P}}^{\mathrm{ion}}\left(\overrightarrow{\mathrm{r}}-\overrightarrow{\mathbf{R}}_{j}+\vec{\tau}\right)\right] \\
& +V_{\mathrm{es}}+V_{\mathrm{xc}}
\end{aligned}
$$

where $\overrightarrow{\mathbf{R}}_{j}$ are the lattice sites of a face-centered-cubic lattice, $\tau$ is the vector connecting a $\mathrm{Ga}$ site to a nearestneighbor $\mathrm{P}$ site, and $v_{\mathrm{Ga}}^{\text {ion }}$ and $v_{\mathrm{P}}^{\text {ion }}$ are ionic pseudopotentials of $\mathrm{Ga}^{3+}$ and $\mathrm{P}^{5-}$, respectively. $V_{\text {es }}$ and $V_{\mathrm{xc}}$ are the self-consistent electrostatic and exchange-correlation potentials, both of which depend on the charge density $\rho^{0}(r)$,

$$
\begin{aligned}
& V_{\mathrm{es}}(\overrightarrow{\mathrm{r}})=\int d^{3} r \frac{\rho^{0}\left(\overrightarrow{\mathrm{r}}^{\prime}\right)}{\left|\overrightarrow{\mathrm{r}}-\overrightarrow{\mathrm{r}}^{\prime}\right|}, \\
& V_{\mathrm{xc}}(\overrightarrow{\mathrm{r}})=\beta\left(\rho^{0}(\overrightarrow{\mathrm{r}})\right) \frac{2}{\pi}\left[3 \pi^{2} \rho^{0}(\overrightarrow{\mathrm{r}})\right]^{1 / 3},
\end{aligned}
$$

where for $\beta\left(\rho^{0}(r)\right)$ we use the analytic form of Hedin and Lundqvist. ${ }^{18}$

The above prescription for the one-electron exchangecorrelation potential is based on the local-density approximation. ${ }^{15}$ This approximation is generally good for the ground-state properties of many-electron systems, but fails to describe the band gap of semiconductors correctly. ${ }^{19}$ The main objective of the present work, however, was to determine the localized states in the region of the gap. Consequently, it was important to adopt a one-electron Hamiltonian which yields a band gap in agreement with the known experimental value, because the energies and character of defect-induced gap states can depend crucially on the energies of the band edges. We proceeded as follows. At first, we constructed ionic pseudopotentials for $\mathrm{Ga}$ and $\mathbf{P}$ by requiring that (a) the free-atom valence energy levels are reproduced, and (b) the atomic valence wave functions are reproduced beyond a certain radius. Using these potentials in a self-consistent band-structure calculation, we obtained a band gap which was about $30 \%$ too small. Furthermore, these calculations failed to reproduce the correct structure of the conduction-band minima, i.e., the minimum at $L$ was below the minimum at $X$, in contrast to experimental results. We, therefore, modified these atom-derived pseudopotentials in order to obtain a satisfactory bulk $\mathrm{GaP}$ band structure. We started with the self-consistent crystal pseudopotential obtained with the atom-derived ionic pseudopotentials and divided it into "crystal-atom" pseudopotentials as follows:

$$
V(\overrightarrow{\mathbf{r}})=\sum_{\overrightarrow{\mathbf{R}}_{j}}^{\mathrm{fcc}}\left[v_{\mathrm{Ga}}\left(\overrightarrow{\mathbf{r}}-\overrightarrow{\mathbf{R}}_{j}\right)+v_{\mathrm{P}}\left(\overrightarrow{\mathbf{r}}-\overrightarrow{\mathbf{R}}_{j}-\vec{\tau}\right)\right] .
$$

The crystal atom pseudopotentials were then modified so that a sufficiently accurate band structure was achieved. The resulting pseudopotentials are listed in Table I. The valence charge density $\rho^{0}(\overrightarrow{\mathrm{r}})$ calculated with these potentials give the electrostatic potential $V_{\mathrm{es}}$ and the local-

TABLE I. Crystal-atom pseudopotentials used in the calculation of the perfect crystal $\left(c_{1} e^{-\alpha_{1} r^{2}}+c_{2} e^{-\alpha_{2} r^{2}}\right)$. The $\alpha$ 's are in (a.u. $)^{-2}$ and the $c$ 's are in rydbergs.

\begin{tabular}{lrr}
\hline & $v_{\mathrm{Ga}}$ & \multicolumn{1}{c}{$v_{\mathrm{P}}$} \\
\hline$c_{1}$ & -20.4 & -17.6 \\
$c_{2}$ & +21.1 & +20.2 \\
$\alpha_{1}$ & 0.45 & 0.45 \\
$\alpha_{2}$ & 0.60 & 0.60 \\
\hline \hline
\end{tabular}


density exchange-correlation potential $V_{\mathrm{xc}}$. The crystal ionic pseudopotential $V^{\text {ion }}$ is then defined by

$$
V^{\mathrm{ion}}(\overrightarrow{\mathrm{r}})=V(\overrightarrow{\mathrm{r}})-V_{\mathrm{es}}(\overrightarrow{\mathrm{r}})-V_{\mathrm{xc}}(\overrightarrow{\mathrm{r}}) .
$$

The localized central-cell parts of the "crystal-ion" pseudopotentials are then defined by subtracting the appropriate superposition of Coulombic tails. Thus, in Rydberg units,

$$
V^{\text {ion }}(\overrightarrow{\mathrm{r}})-\sum_{\overrightarrow{\mathrm{R}}_{j}}\left[\frac{-2 Z_{\mathrm{Ga}}}{\left|\overrightarrow{\mathrm{r}}-\overrightarrow{\mathbf{R}}_{j}\right|} \operatorname{erf}\left(\frac{\left|\overrightarrow{\mathrm{r}}-\overrightarrow{\mathbf{R}}_{j}\right|}{R_{0}}\right)+\frac{-2 Z_{\mathrm{P}}}{\left|\overrightarrow{\mathrm{r}}-\overrightarrow{\mathrm{R}}_{j}-\vec{\tau}\right|} \operatorname{erf}\left(\frac{\left|\overrightarrow{\mathrm{r}}-\overrightarrow{\mathrm{R}}_{j}-\vec{\tau}\right|}{R_{0}}\right)\right)=\left\{\begin{array}{l}
v_{\mathrm{Ga}}^{\text {c.c. }}(\overrightarrow{\mathrm{r}}), \quad 0<|r|<R_{\mathrm{Ga}} \\
v_{\mathrm{P}}^{\text {c.c. }}(\overrightarrow{\mathrm{r}}-\vec{\tau}), \quad 0<|r-\tau|<R_{\mathrm{P}} .
\end{array}\right.
$$

In the above equation, $Z_{\mathrm{Ga}}=3$ and $Z_{\mathrm{P}}=5$. The sum over error functions in Eq. (23) is performed in reciprocal space. For $R_{0}$, we use 3 a.u., for $R_{\mathrm{Ga}}$, we use 3.05 a.u., and for $R_{\mathrm{P}}$, we use 3.34 a.u. The central-cell potentials of Eq. (23) are found to be spherically symmetric within the accuracy of the calculation.

For the band-structure calculation we found that a basis of about 100 plane waves gave a sufficiently accurate description of the energy bands and Bloch functions. The resulting energy bands are shown in Fig. 1. They agree well with the empirical-pseudopotential bands of Chelikowsky and Cohen. ${ }^{20}$ Various transitions are listed in Table II and compared to the experimental values.

The valence charge density of crystalline $\mathrm{GaP}$ is displayed in Fig. 2. We note that the $\mathrm{Ga}-\mathrm{P}$ bonds are largely covalent, as reflected by the buildup of charge be-

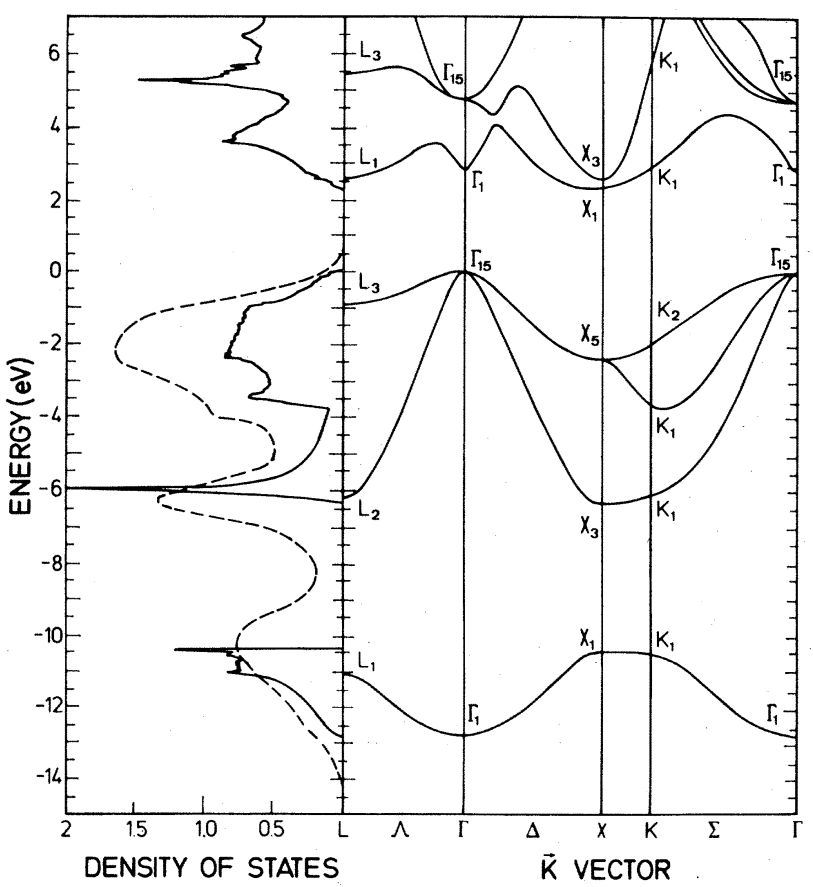

FIG. 1. Calculated band structure for $\mathrm{GaP}$ (right-hand side) and corresponding density of states (left-hand side, solid curve). The dashed curve is the photoemission spectrum from L. Ley, R. A. Pollak, F. R. McFeely, S. P. Kowalczyk, and D. A. Shirley [Phys. Rev. B 9,600 (1974)]. tween nearest neighbors, but they are also partly ionic, as reflected by the fact that the charge in the bond regions is displaced toward the more electronegative $\mathbf{P}$ atoms.

For the defect calculations, we used a localized basis set consisting of $s, p, d$, and $f_{m=0}$ functions centered on the central site and the four nearest neighbors. The radial functions were chosen to be Gaussians of the form $r^{l} e^{-\alpha_{i} r^{2}}$. Three different radial functions $\left(\alpha_{i}=0.1,0.3\right.$, and 0.8 a.u.) were used for $s$ and $p$ functions, and two different radial functions $\left(\alpha_{i}=0.1\right.$ and 0.3 a.u.) were used for $d$ and $f_{m=0}$ functions. The calculations were repeated with additional radial functions (up to five), but the results were essentially the same.

The basis functions were symmetrized in the same manner described in Ref. 7 and were orthogonalized by diagonalizing the overlap matrix. The spectral-density functions [Eq. (17)] were calculated using the GilatRaubenheimer technique ${ }^{21}$ with $203 \overrightarrow{\mathrm{k}}$ points in the irreducible wedge of the Brillouin zone. The real parts of the Green's functions [see Eq. (16)] were calculated by fast Hilbert transforms as in Ref. 7 by using only the first 20 bands. Additional bands were included in test calculations, but their contribution was found to be insignificant for the defects studied in this paper (also see Ref. 22).

The rest of the calculation proceeded in a manner identical to that described in Ref. 7. The only difference was caused by the presence of a sharp resonance-antiresonance pair near the top of the lower s-like valence bands (see Sec. III A) or at the bottom of the $s p$-like valence bands (see Sec. III B).

TABLE II. Interband transitions in $\mathrm{GaP}$ (in $\mathrm{eV}$ ).

\begin{tabular}{lccc}
\hline \multicolumn{1}{c}{ Transition } & $\begin{array}{c}\text { This } \\
\text { calculation }\end{array}$ & Experiment & $\begin{array}{c}\text { Chelikowsky } \\
\text { and Cohen }\end{array}$ \\
\hline$\Gamma_{15}-\Gamma_{1}$ & 2.83 & $2.89^{\mathrm{a}, \mathrm{b}}$ & 2.88 \\
& & $2.97^{\mathrm{a}, \mathrm{b}}$ & \\
$\Gamma_{15}-\Gamma_{15}$ & 4.73 & $5.19^{\mathrm{a}}$ & 5.24 \\
$L_{3}-L_{1}$ & 3.51 & $3.79^{\mathrm{a}}$ & 3.89 \\
$L_{3}-L_{3}$ & 6.36 & $6.38^{\mathrm{c}}$ & 6.84 \\
$\Delta_{5}-\Delta_{1}$ & 4.52 & $4.80^{\mathrm{a}}$ & 4.91 \\
{$[$ at $(0.7,0.0,0.0)]$} & & & \\
\hline
\end{tabular}

${ }^{a}$ C. Varea de Alvarez, J. P. Walter, M. L. Cohen, J. Stokes, and Y. R. Shen, Phys. Rev. B $\underline{6}, 1412$ (1971).

${ }^{\mathrm{b}}$ Spin-orbit splitting (not included in the calculations).

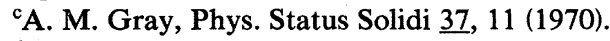

${ }^{\mathrm{d}}$ Reference 16. 


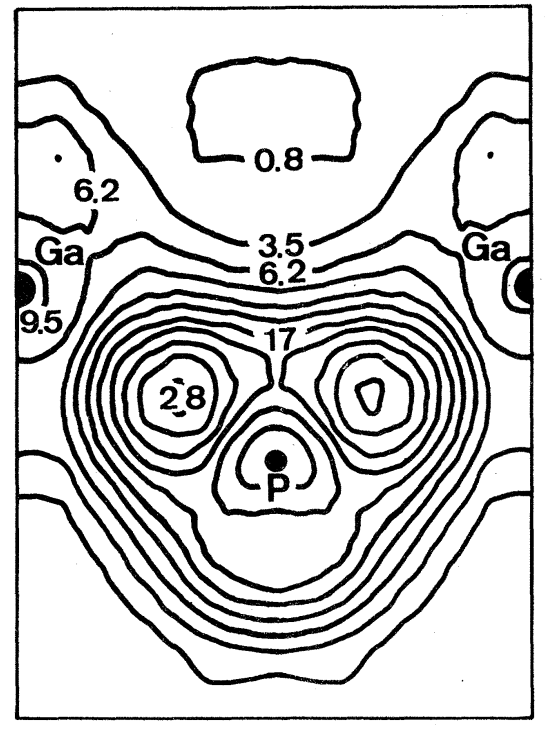

FIG. 2. Calculated valence charge density for $\mathrm{GaP}$ in the (110) plane. The contours are in units of electrons per unit cell.

In order to speed up convergence, we found it expedient to perform the energy integrals in the complex plane. We therefore defined broadened Green's functions by

$$
G^{0}(E)=\left[E-H^{0}+i \Sigma(E)\right]^{-1} .
$$

This energy-dependent broadening does not alter the value of $\rho(\vec{r})$ obtained by integrating over the valence bands and does not therefore represent an approximation. Here, $\Sigma(E)$ was chosen to be $0.3 \mathrm{eV}$ in the region of the valence bands, decaying to zero at the top.

\section{RESULTS}

\section{A. Ga vacancy}

In Fig. 3 we show the calculated density of states of $\mathrm{GaP}$ (top panel) and the changes induced by a $\mathrm{Ga}$ vacancy. As in the case of the vacancy in $\mathrm{Si}^{7,8}$ the most important changes are in the states of $A_{1}$ and $T_{2}$ symmetry. Since the vacancy introduces a repulsive potential corresponding to the removal of an electron-attractive Ga potential, it pushes states to higher energies. In particular, the upper valence bands (from $-7 \mathrm{eV}$ to the top) give rise to a $T_{2}$ bound state in the gap at $E_{v}+0.15 \mathrm{eV}$ and a sharp $A_{1}$ resonance at $E_{v}-0.75 \mathrm{eV}$. (For a neutral vacancy, the $T_{2}$ state in the gap contains three electrons.) The lower valence bands also give rise to a pair of $A_{1}$ and $T_{2}$ localized states both at about $E_{v}-10 \mathrm{eV}$.

Analysis of the charge density associated with the four main localized states reveals that the pair of states in the gap region consist primarily of $p$-like orbitals centered on the nearest neighbors, whereas the pair of states at about $E_{v}-10 \mathrm{eV}$ consist primarily of $s$-like orbitals centered on the nearest neighbors. This result is illustrated for the $T_{2}$ states in Fig. 4. It is consistent with the fact that the upper valence bands are Bloch states consisting primarily of $p$-like states centered on the anions, whereas the lower
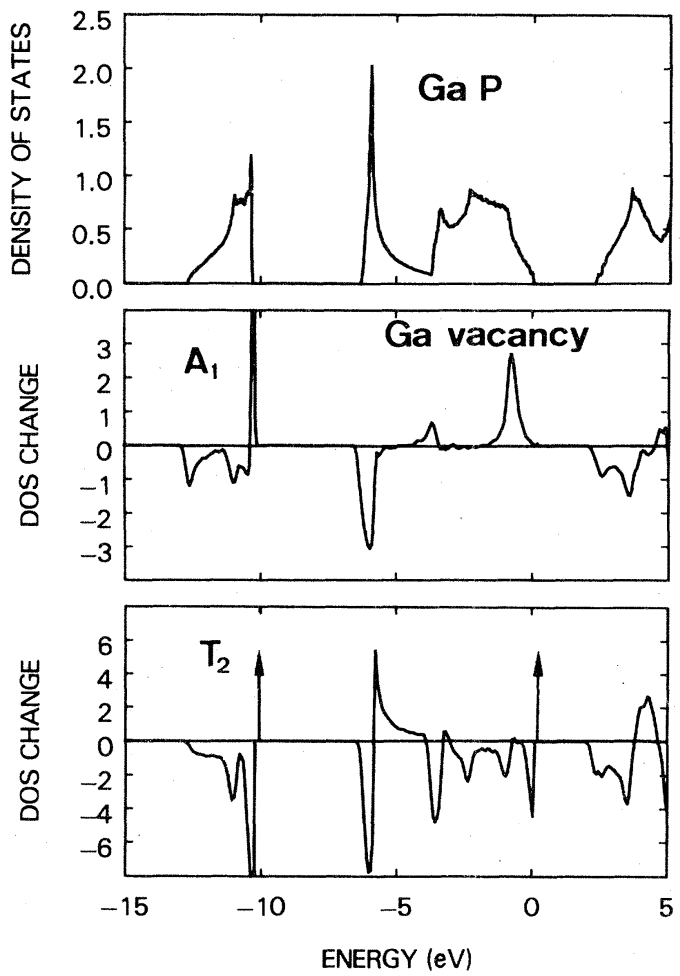

FIG. 3. Density of states (DOS) of GaP (top panel) and the changes in the DOS of $A_{1}$ and $T_{2}$ symmetries induced by a Ga vacancy in $\mathrm{GaP}$.

valence bands are Bloch functions consisting primarily of $s$-like orbitals centered on the anions.

The number of relative energy positions of $A_{1}$ and $T_{2}$ localized states of the Ga vacancy are the same as those of the vacancy in silicon. In the case of the silicon vacancy,
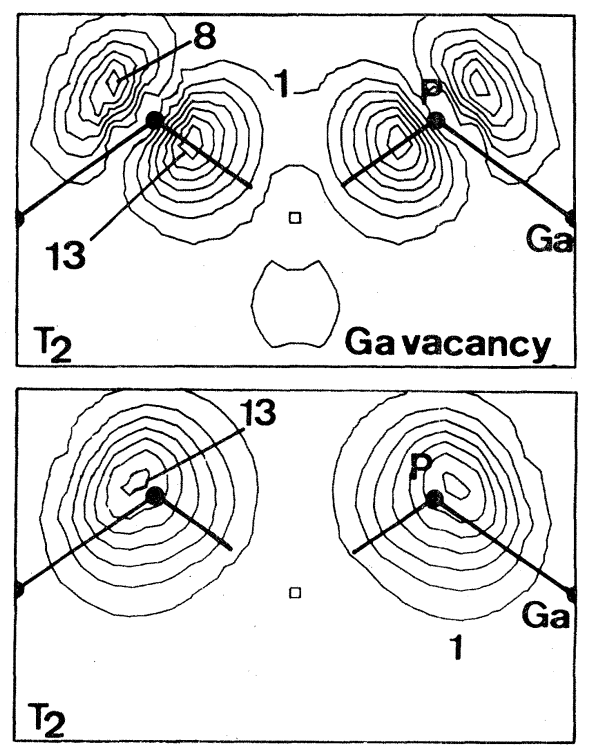

FIG. 4. Charge density in the (110) plane associated with the Ga-vacancy $T_{2}$ localized states at $0.15 \mathrm{eV}$ (top panel) and at $-10.2 \mathrm{eV}$ (bottom panel). Units are $10^{-3}$ electrons (a.u. $)^{-3}$. 
the pair of states in the gap region consists of almost pure $s p^{3}$-like orbitals on the nearest neighbors. In contrast, as we saw above, the corresponding states of the Ga vacancy consist of almost pure $p$-like orbitals centered on the nearest neighbors. This is a significant result because, in the past, deviations from $s p^{3}$ character obtained from analysis of EPR data were often attributed to symmetric, breathing-mode relaxations of the surrounding lattice. ${ }^{2}$ Our calculations demonstrate that deviations from $s p^{3}$ character are possible without breathing-mode relaxation. This result can be understood by noting that the formation of $s p^{3}$ hybrids in the bulk semiconductor is a consequence of the fact that each atom is surrounded by four neighbors in tetrahedral directions. The atoms surrounding a vacancy, however, have only three neighbors, which, even though they are in tetrahedral directions, do not necessarily require the formation of $s p^{3}$ hybrids. More specifically, we can view the localized states of a vacancy as arising primarily from atomic $s$-like and $p$-like orbitals centered on the nearest neighbors. If we symmetrize these orbitals about the vacancy site, we obtain two $A_{1}$ orbitals (the symmetric combination of the $s$ orbitals and the symmetric combination of the $p$ orbitals pointing toward the vacant side) and three $T_{2}$ orbitals (one from the $s$ orbitals and two from the $p$ orbitals). No symmetry requirement determines the ratio of $s$ and $p$ admixture in each of these orbitals. Instead, the ratio must be determined by a calculation and depends on the defect potential and whether or not additional basis orbitals are included. If a calculation were to be performed by including only the above two $A_{1}$ and three $T_{2}$ basis orbitals, we would get two $A_{1}$ and three $T_{2}$ states. Indeed, the full calculation reveals that the vacancy spectrum is dominated by two localized $A_{1}$ states (at -10.4 and $-0.75 \mathrm{eV}$; see Fig. 3) and two $T_{2}$ states (at $-10.2,-5.5$, and $+0.15 \mathrm{eV}$; see Fig. 3 ). The wave functions of these states contain, of course, contributions from basis orbitals centered on additional shells of atoms. These contributions determine the long-range tail of the wave functions.

We note that the ordering of the $A_{1}$ and $T_{2}$ localized states in the gap region are the same for the Ga vacancy and the Si vacancy, namely the $T_{2}$ state lies above the $A_{1}$ state. This ordering is not fixed by any symmetry requirements. As we saw above, however, both these states consist almost entirely of $p$-like orbitals centered on the nearest neighbors. The $A_{1}$ state is the fully symmetric combination of the $p$-like orbitals pointing in the direction of the vacant site and the $T_{2}$ state is a combination of the same $p$-like orbitals with alternative positive and negative signs. Thus, the $A_{1}-T_{2}$ ordering may be viewed as a manifestation of the general result that fully symmetric states usually lie lower in energy than other states constructed from the same orbitals.

In 1978, Kennedy and Wilsey ${ }^{3}$ carried out EPR measurements on electron-irradiated GaP samples and detected a signal which was later labeled NRL-1. This signal was found to have an isotropic $g$ tensor and a center of symmetry which is independent of the direction of the magnetic field, suggesting that the defect responsible for it has full tetrahedral symmetry. Furthermore, the signal had five lines with intensity ratios of $1: 4: 6: 4: 1$ which have

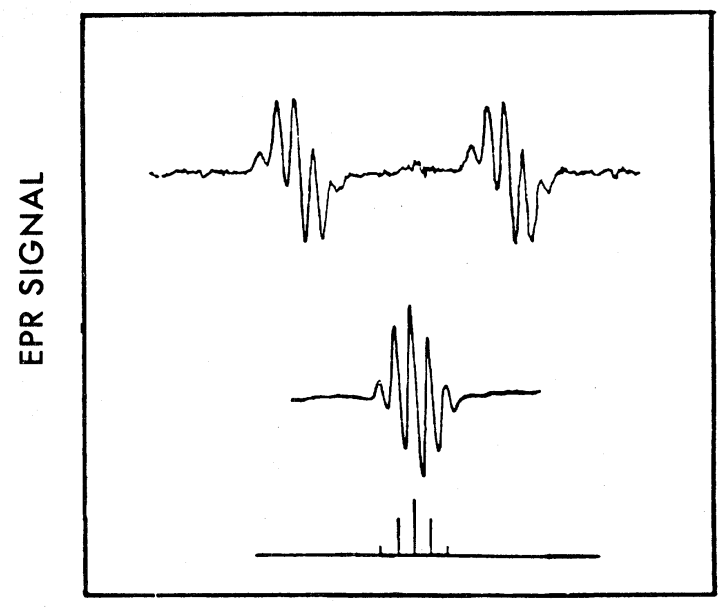

MAGNETIC FIELD

FIG. 5. EPR signal of the $P$ antisite defect (top, from Ref. 1) and the EPR signal labeled NRL-1 (bottom, from Ref. 3). The scale is not the same in the two cases.

been known to be characteristic of an unpaired (spin- $\frac{1}{2}$ ) electron interacting with four spin- $\frac{1}{2}$ nuclei. ${ }^{1}$ In particular, the characteristic five-line structure is present in the spectrum of the $P$ antisite defect ${ }^{1}$ as seen in Fig. 5. The only difference between the $P$ antisite spectrum and NRL-1 is that the antisite spectrum consists of two fiveline structures, whereas NRL-1 consists of only one such structure (Fig. 5). Since the presence of two structures in the antisite spectrum is due to hyperfine interactions with the nuclear spin of $\frac{1}{2}$ of the $P$ atom at the origin, it was concluded $^{3}$ that NRL-1 arises from a single unpaired electron ( $\operatorname{spin} \frac{1}{2}$ ) bound at a center with nuclear spin zero at the origin and four nearest-neighbor ${ }^{31} \mathbf{P}$ atoms in tetrahedral directions. A detailed fit of the data with a standard spin Hamiltonian confirmed this conclusion.

In Ref. 3 three possibilities were considered which were consistent with the above conclusion: (a) a Ga vacancy, (b) a substitutional impurity with nuclear spin zero, and (c) an impurity with nuclear spin zero occupying a tetrahedral interstitial site surrounded by four $\mathbf{P}$ atoms. Impurities were then ruled out and the signal was attributed to the Ga vacancy for the following reasons: (a) the signal is observed only in irradiated material and its intensity increases with electron fluence; (b) a simple model calculation suggested that the wave function had substantial $p$-like character, signaling dangling bonds; (c) the direction and magnitude of the shift in the $g$ value from the freeelectron value is consistent with $g$ shifts of other centers involving dangling bonds observed earlier in $\mathrm{Si}$ and $\mathrm{ZnSe}$; (d) all the possible impurities that were present in their samples were identified and ruled out for different reasons.

One potential difficulty was noted in Ref. 3 with the assignment of NRL-1 to the Ga vacancy. The vacancy in Si had been identified earlier and was known to have reduced symmetry because of Jahn-Teller distortions. These distortions occur because the Si vacancy has a partially occupied sixfold-degenerate $T_{2}$ level in the gap, above a fully occupied $A_{1}$ level. It was suggested ${ }^{3}$ that the $\mathrm{Ga}$ 
vacancy's apparent full tetrahedral symmetry can be explained if one assumes that the $A_{1}$ and $T_{2}$ levels are in reverse order, namely a fully occupied $T_{2}$ level below a partially occupied $A_{1}$ level. The observed signal would then correspond to the $V^{2-}$ charge state.

The position of the energy level containing the unpaired electron could not be determined very precisely in Ref. 3 . It was noted, however, that the EPR signal was observed in $p$-type material only after photoexcitation with light of frequency larger than $1.7 \mathrm{eV}$ for the entire range of electron fluences. Low electron fluences leave the Fermi level at the shallow-acceptor level, whereas increasingly larger fluences compensate the sample and move the Fermi level higher, and eventually to the midgap region. It was therefore concluded that the energy level containing the unpaired electron is either below the shallow-acceptor level or in the upper half of the gap. The additional observation of the EPR signal in $n$-type material without the need of photoexcitation led to the conclusion that the level is in fact in the upper part of the gap.

In 1981, we reported ${ }^{11}$ the results of our theoretical calculations for the $\mathrm{Ga}$ vacancy and challenged the conclusions of Ref. 3. The main result was the ordering of the $A_{1}$ and $T_{2}$ level. We found the $T_{2}$ level consistently above the $A_{2}$ level for all charge states and for both in-

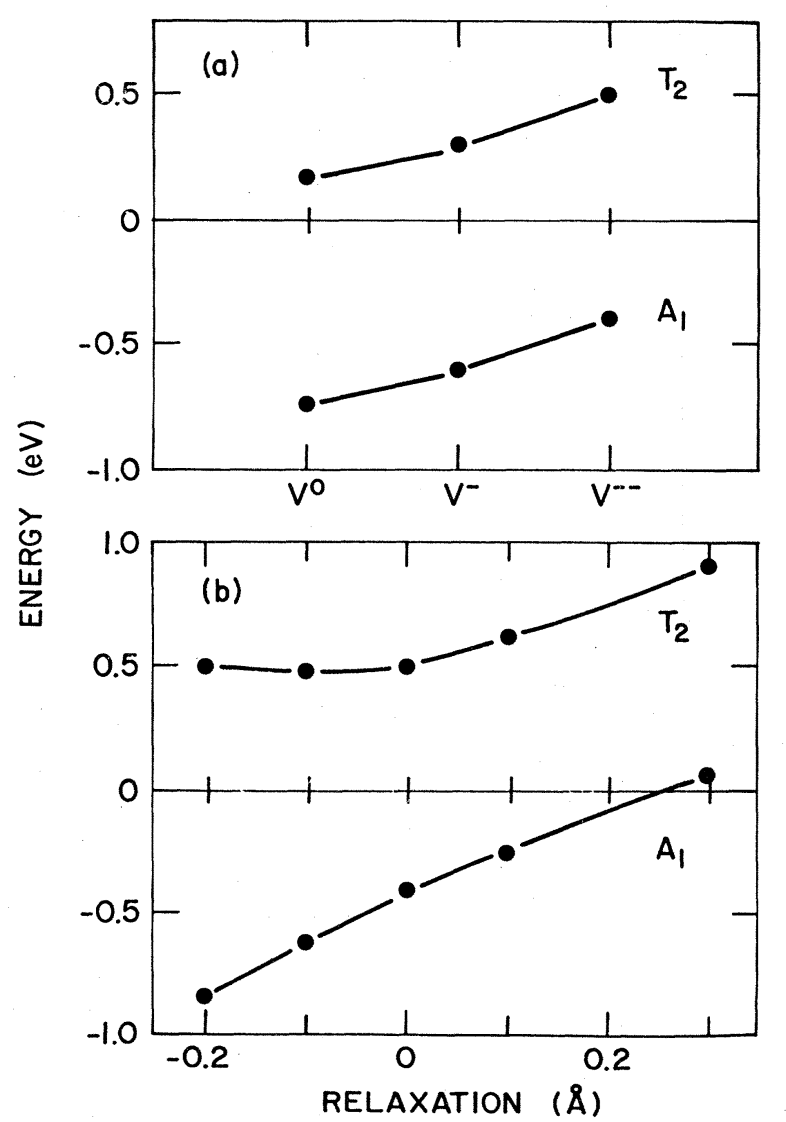

FIG. 6. (a) Variation of the $T_{2}$ and $A_{1}$ localized states of the unrelaxed $\mathrm{Ga}$ vacancy in $\mathrm{GaP}$ for three different charge states. (b) Variation of the same states of the doubly-negatively-charged Ga vacancy as a function of inward and outward symmetric relaxation of the nearest neighbors. ward and outward symmetric relaxations of the nearest neighbors (Fig. 6). Thus, if one places two electrons in the $A_{1}$ level, which lies inside the valence bands, and any odd number of electrons in the $T_{2}$ level in order to have a single unpaired electron and a net electronic spin of $\frac{1}{2}$, one has an orbitally degenerate state which must inevitably lead to Jahn-Teller distortions (also see the Appendix). Thus, none of these charge states of the Ga vacancy can have spin $\frac{1}{2}$ and full tetrahedral symmetry. Furthermore, the $T_{2}$ level of all the Ga-vacancy charge states lies in the lower half of the band gap, which is inconsistent with the conclusion arrived from the need to photoexcite the signal in $p$-type but not $n$-type material. ${ }^{3}$ One might also consider the possibility that NRL-1 arises from an excited state of the vacancy, namely a state that has only one electron in the $A_{1}$ level and up to six electrons in the $T_{2}$ level. Such an excited state of $V^{2-}$ would indeed have total electronic spin of $\frac{1}{2}$, would be orbitally nondegenerate and thus stable against Jahn-Teller distortions, and thus appear consistent with the properties of NRL-1. It would not, however, be consistent with the observation that NRL-1 is seen in the dark in $n$-type samples.

In view of the difficulties faced by the notion that NRL-1 is due to Ga vacancies, in Ref. 11 we considered alternative possibilities and determined that carbon impurities at $\mathrm{Ga}$ sites are indeed consistent with all the then available data. ${ }^{3,23}$ We will not discuss the case for carbon further, however, because newer data have resulted in significant changes in the properties attributed to NRL-1, making carbon an unlikely candidate.

Since the publication of Ref. 11, a number of new experiments were carried out to test the two possible identifications of NRL-1. Measurements of the carbon concentration, though potentially promising, did not prove conclusive. ${ }^{24}$ More recently, uniaxial-stress measurements were reported ${ }^{12}$ which led to a reassignment of the NRL-1 signal to a center with a total electronic spin of $\frac{3}{2}$. This reassignment immediately rules out both $V^{2-}$ and $\mathrm{C}_{\mathrm{Ga}}$ because both these centers have total electronic spin of $\frac{1}{2}$. A total electronic spin of $\frac{3}{2}$ is possible only if a sixfolddegenerate level in the gap contains three electrons with parallel spin (see the Appendix). Such a state is orbitally nondegenerate and would, therefore, make the center stable against Jahn-Teller distortions. It was, ${ }^{12}$ therefore, natural to assign the NRL-1 EPR signal to the neutral Ga vacancy which has been shown by our calculations ${ }^{11}$ to have just such a level in the gap. Such an assignment, however, leads to one difficulty not addressed in Ref. 12. The neutral Ga vacancy has its energy level in the lower part of the band gap and the negatively charged vacancy is only about $0.3 \mathrm{eV}$ higher in energy, i.e., still in the lower half of the gap [the value seen in Fig. 6(a) is only about $0.2 \mathrm{eV}$; the value $0.3 \mathrm{eV}$ is obtained if the effect of the Coulombic tail is included by perturbation theory]. Thus, in $n$-type samples, in which the Fermi level lies in the upper half of the band gap, Ga vacancies should exist primarily in negatively charged states. As reported in Ref. 3, however, NRL-1 is seen in the dark, the signal is weak, and photoexcitation has no effect on it. This difficulty with the vacancy identification of NRL-1 has been communicated to the authors of Refs. 3 and 12. We have 
since been informed that the data on $n$-type samples have been reexamined and the report in Ref. 3 was found to be in error. In particular, contrary to what is stated in Ref. 3, photoexcitation does change the signal strength of NRL-1. A more thorough study of the excitation properties of NRL-1 is now under way ${ }^{13}$ and we cannot, therefore, draw firm conclusions at this time.

In 1982, Mooney, Kennedy, and Small ${ }^{14}$ reported DLTS measurements whose purpose was to identify the energy level of the center responsible for NRL-1. They detected two radiation-induced hole traps, one of which at $E_{v}+0.64 \mathrm{eV}$ had the same isochronal annealing temperature and roughly the same introduction rate as NRL-1. From the $T=\infty$ intercept of the Arrhenius plot, they estimated that the capture cross section of this center is $4 \times 10^{-15} \mathrm{~cm}^{2}$, and hence concluded that the level at 0.64 $\mathrm{eV}$ corresponds either to a $0 /-$ or a $-/--$ transition. The annealing-temperature and introduction-rate correlations suggest that the same center is involved in both cases, but one cannot tell if the two experiments probe the same charge state of the center. We note again that the assignment of the $0.64-\mathrm{eV}$ level to NRL-1 would be inconsistent with the published data on $n$-type samples. ${ }^{3}$ Since, however, these data have been revised, ${ }^{13}$ this objection is removed. We note that the $0.64-\mathrm{eV}$ level lies $1.7 \mathrm{eV}$ below the conduction-band edge, which is precisely the energy of the light needed to photoexcite NRL-1 in $p$-type samples. One might then infer that photoexcitation of NRL-1 amounts to converting $V^{-}$to $V^{0}$ by the removal of an electron to the conduction bands. Such a level scheme, however, has a serious shortcoming: It requires that the Fermi level in all irradiated $p$-type samples lies above 0.64 $\mathrm{eV}$. That is impossible, however, because, at low fluences, the Fermi level does not move appreciably from the shallow-acceptor level. . $^{3,14}$

Even more recently, Mooney and Kennedy ${ }^{15}$ measured the capture cross section of the DLTS level directly. They report a new value about 2 orders of magnitude smaller, i.e., somewhere between $4 \times 10^{-17}$ and $9 \times 10^{-17} \mathrm{~cm}^{2}$. This value implies capture by a neutral center and leads to the assignment of the $0.64-\mathrm{eV}$ level to a $+/ 0$ transition. ${ }^{15}$ One can therefore assign the 0.64-eV DLTS level to the $+/ 0$ thermal level of the Ga vacancy. With this level structure, however, the photoexcitation data are more difficult to understand. Since the EPR signal is not seen in any irradiated $p$-type samples without photoexcitation, one can conclude that the Fermi level remains below $E_{v}+0.64 \mathrm{eV}$ even after high irradiation fluences, contrary to the original assessment of Ref. 3. One possible explanation for this effect is pinning of the Fermi level by another irradiation-induced level, for example, the $I_{1}$ level observed by DLTS by Mooney et al. ${ }^{14}$ at $E_{v}+0.51 \mathrm{eV}$. With the Fermi level below $E_{v}+0.64 \mathrm{eV}$, the Ga vacancy exists in a $V^{+}$charge state which can have spin 0 (see the Appendix) and thus be EPR inactive. A look at the possible spin-0 multiplets of $V^{+}$(see Appendix) reveals that the most likely ground state is orbitally degenerate, which triggers a Jahn-Teller distortion. Whatever the mode of distortion, the result is probably a splitting of the $T_{2}$ level so that a singlet contains two electrons, one with spin up and one with spin down, for a total spin of 0 , very much like the neutral state of the vacancy in $\mathrm{Si}^{25}$

We turn now to the photoexcitation process itself. It was suggested in Ref. 3 that photoexcitation occurs indirectly, i.e., electrons are excited from some other unknown trap to the conduction bands and are then captured by the primary center, in this case the Ga vacancy, which becomes EPR active. Such an indirect process can explain the observed slow photoexcitation (about two hours ${ }^{3}$ ). This process is highly unlikely, however, because, as we saw above, in all the $p$-type samples the Fermi level remains low in the band gap. Under low electron fluences, the Fermi level in fact remains at the shallowacceptor level. ${ }^{23}$ Thus, there exist no traps containing electrons which can be photoexcited to the conduction bands with photons of energy only $1.7 \mathrm{eV} .^{26}$ We therefore consider the possibility that photoexcitation is direct, i.e., an electron is directly excited from the valence bands to one of the gap states of the positively charged vacancy, converting it to the spin- $\frac{3}{2}$ EPR-active neutral vacancy. We have already remarked that $V^{+}$most likely has two electrons with opposite spins in a singlet level which is the low-energy member of the Jahn-Teller-split $T_{2}$ state in the gap. We note that direct photoexcitation must involve exciting an electron from the valence bands to a higherenergy member of the split $T_{2}$ state. Thus, by direct photoexcitation, one cannot access the spin- $\frac{3}{2}$ state of the neutral vacancy which has all three electrons parallel. Optically, one can access only a spin- $\frac{1}{2}$ state, which can subsequently convert to the spin- $\frac{3}{2}$ state by flipping the spin of one of the electrons. The spin flip can be accomplished over a period of time by spin-orbit interactions which have been left out of the analysis so far and hence act as perturbations. ${ }^{27}$ This process can explain the slowness of the photoexcitation, but raises new questions. First, the initial spin- $\frac{1}{2}$ state should be EPR active, yet there is no evidence that NRL-1 builds up as another EPR signal is reduced. One could explain this difficulty by arguing that photoexcitation creates spin- $\frac{1}{2}$ neutral vacancies which decay fast by direct recombination except for a fraction that succeed in converting to the spin- $\frac{3}{2}$ state, which is more stable. At any given time the concentration of spin- $\frac{1}{2}$ vacancies may be too small to detect by EPR, so that an EPR signal is not detected until a sizeable concentration of spin- $\frac{3}{2}$ centers is built up. A final difficulty remains with this model, however; namely the size of the minimum photon energy required for photoexcitation, 1.7 $\mathrm{eV}^{3}$ Our calculations find the neutral vacancy level at $E_{v}+0.15 \mathrm{eV}$. Even with breathing-mode relaxation, the $T_{2}$ level of $V^{+}$must be very near the bottom of the gap. Jahn-Teller distortions would split this level, the occupied singlet may enter the valence bands, but the empty members of the split level are unlikely to move up by more than a few tenths of an $\mathrm{eV}$ (compare with results for the vacancy in Si, Ref. 25). A value of $1.7 \mathrm{eV}$ is much too large. Even if one allows for weak oscillator strengths for excitations from the first few tenths of an $\mathrm{eV}$ of the valence bands, a substantial discrepancy still exists.

It is evident from the above analysis that a complete and detailed picture has not yet emerged. Let us, however, summarize what we have learned so far. We note that 
the key experimental observation is that the NRL-1 EPR spectrum has the characteristic five-line structure that suggests a center containing four $\mathbf{P}$ atoms in tetrahedral positions from an origin. NRL-1 differs from the spectrum of the $\mathbf{P}$ antisite defect in that the latter exhibits hyperfine splitting by a nuclear spin of $\frac{1}{2}$ at the central site, whereas NRL-1 does not (Fig. 5). One then concludes that NRL-1 originates from a center with four $\mathbf{P}$ atoms in tetrahedral positions about a site with nuclear spin 0 . That much seems to be beyond any doubt and, with that much information, both the Ga vacancy and carbon at $\mathrm{Ga}$ sites are viable candidates. In order to differentiate between the two, one needs to determine the total electronic spin of the center and have some knowledge about the electronic energy levels and the two candidate centers. The recent uniaxial-stress measurements ${ }^{12}$ rule in favor of spin $\frac{3}{2}$, which makes the Ga vacancy the only viable candidate. There is a noteworthy observation here, namely that the key elements for the identification of NRL-1 as the neutral Ga vacancy are (1) its similarity with the EPR spectrum of the $P$ antisite defect (Fig. 5) that allows one to conclude that NRL-1 has four $\mathbf{P}$ atoms about a center with nuclear spin 0, and (2) its difference from the EPR spectrum of the $\mathbf{P}$ antisite defect that allows one to conclude that NRL-1 has total electronic spin $\frac{3}{2}$ instead of $\frac{1}{2}$. This difference has not, in fact, been established, since uniaxial-stress data have been reported only for NRL-1. It is, therefore, important that the EPR spectrum of the P antisite defect is studied under the same uniaxial-stress and temperature conditions to establish this key difference of electronic spin. In addition, the value of the electronic spin for each center can be established independently and unambiguously by electron nuclear double-resonance (ENDOR) measurements.

We conclude that the recent uniaxial-stress measurements constitute strong evidence that NRL-1 arises from the spin- $\frac{3}{2}{ }^{4} A_{2}$ state of the neutral Ga vacancy. Additional experiments are needed, however, to establish the value of the spin independently and confirm the identification. As for the energy-level structure of this center, there appear to be discrepancies between the theoretical levels of the vacancy and the level structure suggested by photoexcitation and DLTS data. We note, however, that the data are far from complete, some published data have been revised in private communications, and many gaps remain. It is therefore impossible to try to resolve the existing discrepancies until systematic data on the photoexcitation requirements of NRL-1 in both $n$-type and $p$-type samples are published. At the same time, theoretical calculations of the multiplets and of the effect of symmetry-breaking Jahn-Teller distortions would be necessary for a systematic comparison between theory and experiment. For example, the equilibrium spin state of the neutral vacancy is determined by balancing the energy gained from aligning the spins of the three electrons in the gap state without Jahn-Teller distortions against the energy gained from Jahn-Teller distortions which accompany spin pairing. Such calculations are not feasible at present without parametrizing some interactions, but they are necessary to explain, among other things, why spin alignment is favored over Jahn-Teller distortions in the case of the neu- tral Ga vacancy in GaP, but not in the case of $V^{-}$in $\mathrm{Si}$, which also has three electrons in the gap. Such calculations would also establish a more accurate energy-level structure to be compared with experimental data.

\section{B. P antisite defect}

The phosphorus antisite $P_{G a}$ is a typical example of a substitutional double donor, i.e., it can compensate two shallow acceptors. A very similar electronic structure is expected for $\mathrm{As}_{\mathrm{Ga}}$ and $\mathrm{Sb}_{\mathrm{Ga}}$. Furthermore, the electronic structure of these centers is expected to be similar to that of the double donors in $\mathrm{Si}$, such as $\mathrm{Si}: \mathrm{S} .{ }^{9,10}$ Figure 7 shows the calculated changes in the density of states due to a $\mathbf{P}$ antisite defect. As expected, since the defect potential is attractive, state density is shifted to lower energies and band states appear below band edges. The number, relative energy positions, and orbital content of the localized states can be understood in terms of simple physical models, similar to those employed in Ref. 10 for impurities in Si. In Fig. 8 we show the charge density of the three $A_{1}$ bound states. These states can be understood in terms of the following two different, but related, models.

(a) The bound-state wave functions may be viewed as consisting primarily of $s$ and $p$ orbitals centered on the "impurity" (i.e., the central $P$ atom) and its four nearest neighbors. The orbitals on the neighbors can be symmetrized into orbitals of $A_{1}$ and $T_{2}$ symmetry. Thus, $A_{1}$
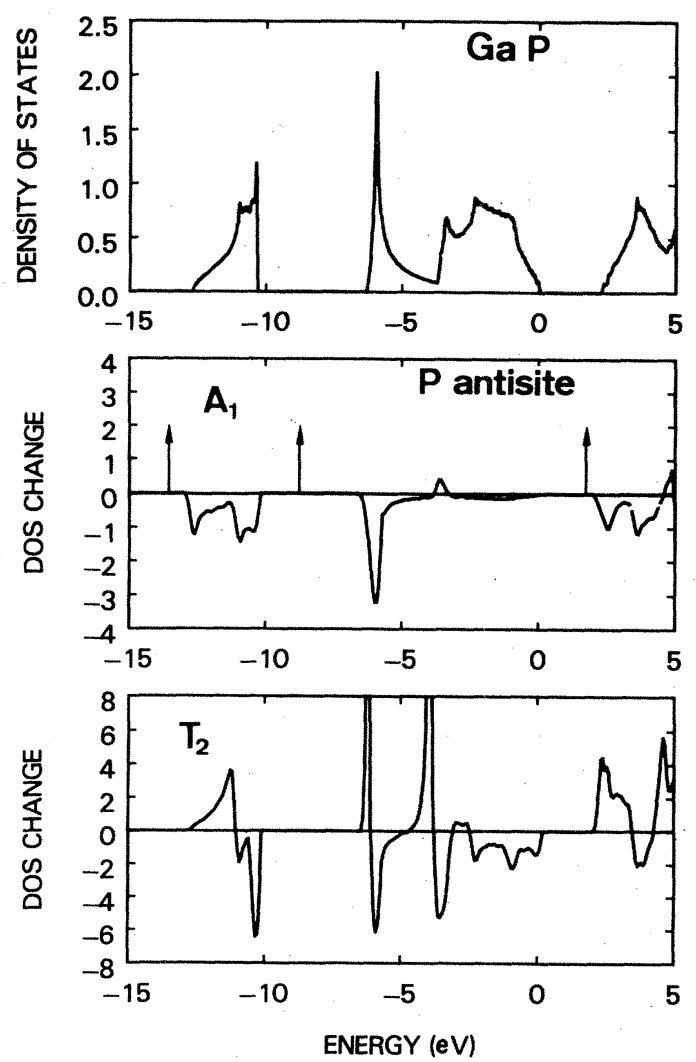

FIG. 7. Perfect-crystal density of states of GaP (top panel) and changes induced in the density of $A_{1}$ and $T_{2}$ states by a phosphorus antisite defect. 

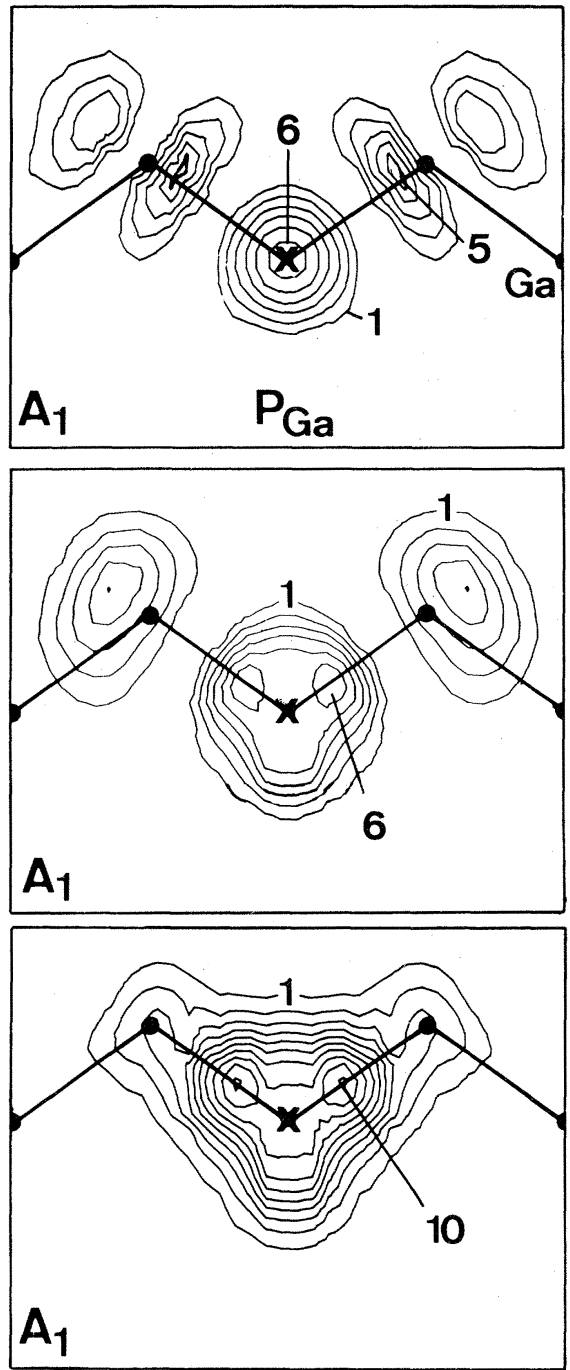

FIG. 8. Charge densities associated with the three $A_{1}$ localized states induced by a phosphorus antisite defect in GaP. Top panel: state at $1.9 \mathrm{eV}$; middle panel; state at $-8.5 \mathrm{eV}$; bottom panel: state at $-13.5 \mathrm{eV}$.

localized states can be formed from the following basis orbtials:

$$
\phi_{\alpha}=s_{I}, \quad \phi_{\beta}=N_{s} \sum_{n} s_{n}, \phi_{\gamma}=N_{p} \sum_{n} p_{n},
$$

where $s_{I}$ is the impurity $s$ orbital, $s_{n}$ is the $s$ orbital centered on the $n$ th-nearest neighbor, $p_{n}$ is the $p$ orbital centered on the $n$ th-nearest neighbor pointing in the direction of the impurity, and $N_{s}, N_{p}$ are normalization constants. For donors the ordering of energies of these orbitals is shown in Fig. 9 (left and right). When these basis orbitals are allowed to interact, the states shown in Fig. 9 (middle) follow. The lowest state is bondinglike, consisting primarily of impurity $s$ orbitals, and the middle state is the corresponding antibonding orbital, consisting primarily of $s$ orbitals on the impurity and nearest neighbors. Finally, the highest state is also antibondinglike, consisting primarily of $p$ orbitals on the nearest neighbors and a small admixture of $s$ orbitals. These results are consistent with

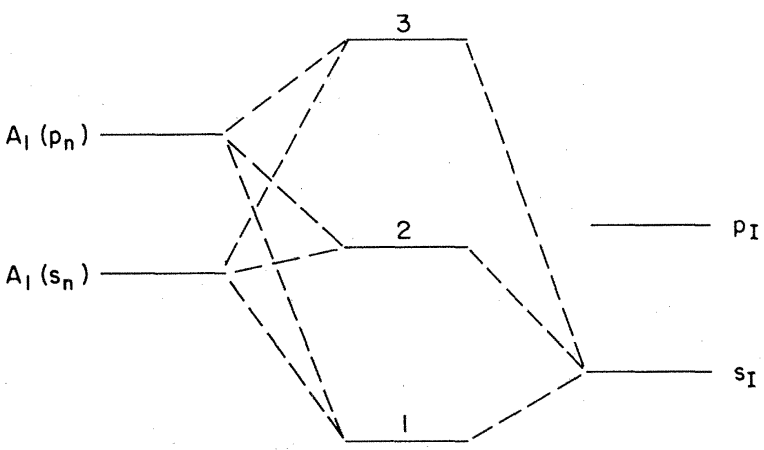

FIG. 9. Schematic illustration of how the $s$ orbital of the central impurity atom $\left(s_{I}\right)$ and the $A_{1}$ linear combinations of the $s$ and $p$ orbitals of the host nearest-neighbor atoms interact to give rise to the localized states associated with a $\mathrm{P}$ antisite defect of any deep donor impurity.

the actual wave functions shown in Fig. 8. A similar picture can be constructed for $T_{2}$ states. In general, however, such models are only interpretive, not predictive, since one or more of the states resulting from these models may end up being degenerate with a high density of crystal states, becoming a broad resonance and thus not recognizable as a localized state.

(b) The bound states may be viewed as arising from interactions between the $s$ and $p$ states of the free impurity atom (in this case a $\mathbf{P}$ atom) with the localized states of a vacancy. This point of view is closely related to the one described above because the localized states of a vacancy can be understood as consisting predominantly of $s$ and $p$ orbitals centered on the four nearest neighbors. For example, the Ga vacancy has two localized $A_{1}$ states in the valence-band region, one at $E_{v}-0.75 \mathrm{eV}$ and one at $E_{v}-10.4 \mathrm{eV}$. These two states consist predominantly of a mixture of the orbitals $\phi_{\beta}$ and $\phi_{\gamma}$ defined above. In particular, the high-energy state contains mostly $p$ orbitals of the nearest neighbors and the lower-energy state contains mostly $s$ orbitals of the nearest neighbors. When these two states are allowed to interact with the $s$ state of the impurity, they give rise to three new states which are precisely the ones shown in Figs. 6 and 7. Thus the state in the fundamental gap may be viewed as derived from the vacancy $A_{1}$ state just below the valence-band edge, pushed up by interacting with the impurity-atom $s$-like state.

The changes in the density of $T_{2}$ states can be understood in a similar way. The $3 p$ state of a $\mathbf{P}$ atom interacts with the three $T_{2}$ localized states of the Ga vacancy (at $-10.2,-5.5$, and $+0.15 \mathrm{eV})$ and gives rise to four new localized states seen in Fig. 7 at $-12.0,-6.5,-4.0$, and $+2.5 \mathrm{eV}$. We note that in both the $A_{1}$ and $T_{2}$ symmetry we do not have a simple two-level system as proposed by Hjalmarson et al. in their recent tight-binding model of deep impurities. ${ }^{28}$ As illustrated by the above discussion for the antisite defect, the actual picture is, in fact, considerably more complicated.

The $\mathbf{P}$ antisite defect has been identified in as-given material by Kaufmann et al. using EPR. ${ }^{1}$ The observed spectra are due to an unpaired electron in a localized state with effective spin $\frac{1}{2}$. The nuclear spin of the central 


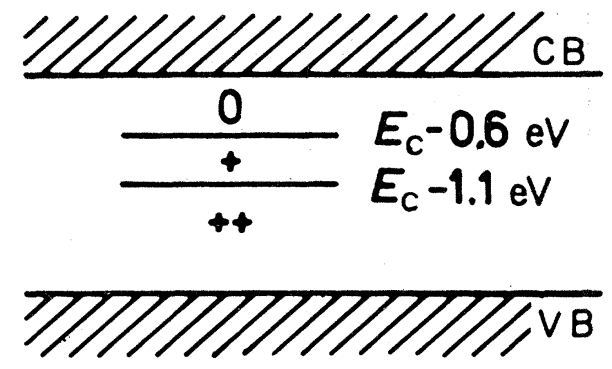

FIG. 10. Three charge states of a $\mathrm{P}$ antisite defect in $\mathrm{GaP}$ in the absence of lattice relaxations.

atom and that of the four neighbor atoms is $\frac{1}{2}$. The symmetry has been determined as $T_{d}$. Because $\mathrm{P}$ is the only atom with a nuclear spin $\frac{1}{2}$ present in the sample with sufficiently high concentrations, these EPR spectra are a "fingerprint" of a $\mathbf{P}$ antisite defect. The fact that the hyperfine splitting due to the neighboring nuclei is clearly resolved reflects a high degree of localization of the EPRactive wave function. In semi-insulating material the EPR signal is observed in the dark, and the signal is reduced if light of energy higher than $0.85 \mathrm{eV}$ is applied. In $p$-type material the center is not EPR active in the dark, i.e., the responsible deep level is empty. Light of energy higher than $1.25 \mathrm{eV}$, however, excites electrons from the valence band into the empty level and EPR is observed. These experimental results are consistent with our theoretical results for the wave function in the gap (top of Fig. 8), which show that the wave function is indeed highly localized on the central atom and the nearest neighbors. The antibonding character of this wave function implies that the binding between the central $\mathrm{P}$ atom and its nearest neighbors is strongest if the deep level is empty. This charge state is realized in $p$-type samples when the center is in a $2+$ state. As the Fermi level is raised and the level in the gap becomes occupied by one or two electrons, the binding forces between the central $\mathbf{P}$ atom and its neighbors becomes weaker.

The antisite defect can, in principle, exist in three different charge states, neutral, + , and $2+$. We therefore calculated the ionization energies for the $\mathrm{P}_{\mathrm{Ga}}^{0} \rightarrow \mathrm{P}_{\mathrm{Ga}}^{+}$and the $\mathbf{P}_{\mathrm{Ga}}^{+} \rightarrow \mathbf{P}_{\mathrm{Ga}}^{2+}$ transitions using the transition-state technique and assuming an undistorted lattice. The Coulombic tail which is present in these transition states is included by perturbation theory as $-n(0.1 \mathrm{eV})$ with $n=\frac{1}{2}$ and $\frac{3}{2}$, respectively. The theoretical numbers for the first and second ionization energies are 0.6 and $1.1 \mathrm{eV}$ (Fig. 10). They agree well with the corresponding experimental numbers. i.e., $<0.8$ and $(1.1 \pm 1.0) \mathrm{eV}$, respectively, which were extracted from optical data. ${ }^{5}$ More recently, Ferenc$\mathrm{zi}^{29}$ performed DLTS experiments on plastically deformed $\mathrm{GaP}$ junctions and found two levels, one at $(1.2 \pm 0.05) \mathrm{eV}$ and one at $(0.75 \pm 0.1) \mathrm{eV}$. He attributed these levels to the $\mathbf{P}$ antisite defect. Again these two levels are consistent with our theoretical results.

\section{Carbon on $\mathbf{G a}$ sites $\left(\mathrm{C}_{\mathrm{Ga}}\right)$}

When first-row elements are introduced as substitutional impurities, they are well known to behave differently from the elements of other rows of the Periodic Table. For example, whereas $\mathrm{P}, \mathrm{As}, \mathrm{Sb}$, and $\mathrm{Bi}$ are shallow donors in $\mathrm{Si}^{30} \mathbf{N}$ is known to be deep. ${ }^{31}$ Similarly, whereas $\mathrm{S}, \mathrm{Se}$, and $\mathrm{Te}$ are shallow donors at the $\mathrm{P}$ site in $\mathrm{GaP},{ }^{32} \mathrm{O}$ is deep. ${ }^{33}$ The elements $\mathrm{Si}, \mathrm{Ge}$, and $\mathrm{Sn}$ are known to occupy $\mathrm{Ga}$ sites in GaP and to act as shallow donors. The corresponding first-row element, carbon, has not been detected at a Ga site. Dean et al. ${ }^{36}$ in 1968 suggested that the failure to detect $\mathrm{C}_{\mathrm{Ga}}$ was due to the fact that it is deep because of carbon's high electronegativity. In 1978, Kennedy and Wilsey ${ }^{3}$ assigned their NRL-1 EPR signal to the Ga vacancy by ruling out several impurities with nuclear spin 0 . Carbon, which has 0 nuclear spin, was a possible candidate because it is present in sufficient concentrations to account for the strengths of the observed EPR signal. ${ }^{3}$ It was ruled out, however, on the grounds that it would be a shallow donor and thus have an EPR spectrum and $g$ value similar to those of $\mathrm{Si}, \mathrm{Ge}$, and Sn. When our work on the vacancy (Ref. 11 and Sec. III A) disputed Kennedy and Wilsey's assignment of NRL-1 to $V^{2-}$, we noted that $\mathrm{C}_{\mathrm{Ga}}$ would be consistent with the data if it was deep. The analogy with Si:N and GaP:O$O_{P}$ already suggested that $C_{G a}$ might indeed be deep. We therefore performed self-consistent Green's-function calculations using the nonlocal $\mathrm{C}$ ionic pseudopotential of $\mathrm{Ihm}$, Louie, and Cohen. ${ }^{35}$ We found a deep $A_{1}$ level in the gap whose properties were fully consistent with the properties of NRL-1 as originally reported by Kennedy and Wilsey. ${ }^{3}$ Since then, however, new experiments revealed that NRL-1 originates from a center with total electronic spin of $\frac{3}{2}$, which rules out carbon as a candidate and leaves the neutral vacancy as the most likely candidate for

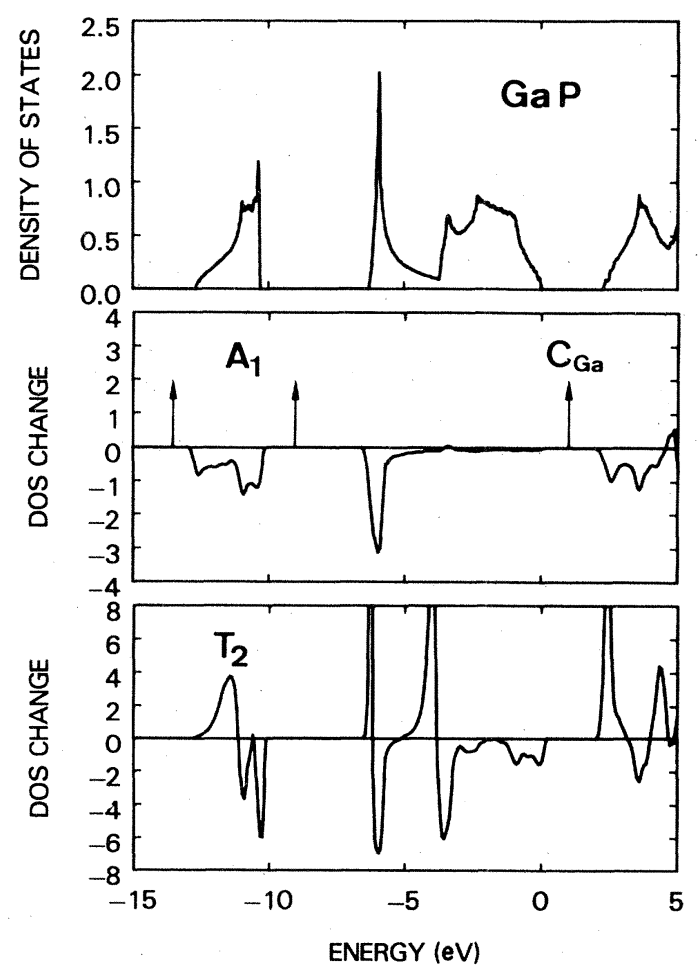

FIG. 11. Perfect-crystal density of states of GaP and the changes induced in the density of $A_{1}$ and $T_{2}$ states by a carbon impurity at a $\mathrm{Ga}$ site. 

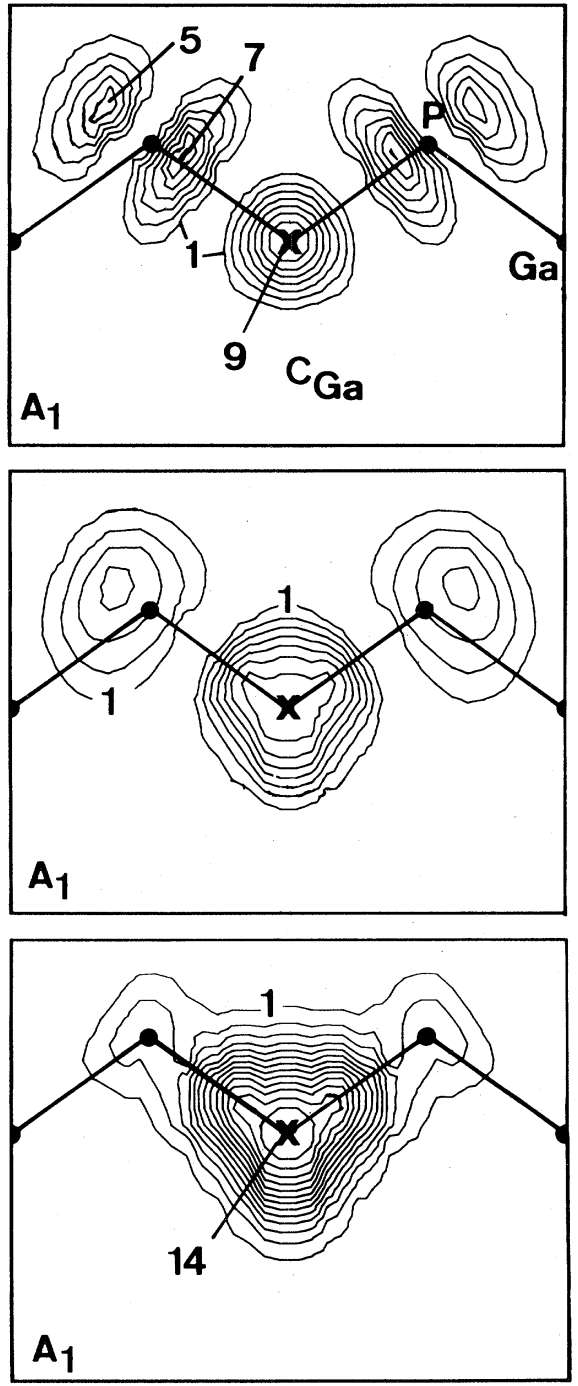

FIG. 12. Charge density associated with the three $A_{1}$ localized states induced by a carbon impurity at a $\mathrm{Ga}$ site in $\mathrm{GaP}$. Units as in Fig. 4.

NRL-1. A full discussion was given in Sec. III A.

Figure 11 shows the calculated changes in the density of states for $\mathrm{C}_{\mathrm{Ga}}$, and Fig. 12 shows the charge density associated with the $A_{1}$ bound states in the gaps. If we compare these figures with the corresponding results for the $\mathbf{P}$ antisite defect (Figs. 6 and 7), we see that these two systems are very similar. Antiresonances and resonances appear at essentially the same energies in the two systems. In particular, antiresonances appear at energy regions where the perfect-crystal state density is high, whereas resonances appear in energy regions where the perfectcrystal state density is low. ${ }^{7}, 36$ Bound states are somewhat deeper in $\mathrm{C}_{\mathrm{Ga}}$, but the corresponding wave functions are very similar in the two systems. Thus, the origin of the bound states of Fig. 11 can be understood in the same way as described for the antisite defect in Sec. III B.

\section{SUMMARY}

In this paper we reported detailed calculations of the electronic structure of cation-site defects in GaP, namely the $\mathrm{Ga}$ vacancy, the $\mathrm{P}$ antisite defect ( $\mathrm{P}$ at a normally $\mathrm{Ga}$ site), and $\mathrm{C}$ at a $\mathrm{Ga}$ site.

The results for the unrelaxed vacancy show that the localized dangling-bond states do not have $s p^{3}$ hybrid character, as is usually assumed. In addition, we find that the localized state of $T_{2}$ symmetry lies above the state of $A_{1}$ symmetry. The consequences of this result have been significant. In 1978, Kennedy and Wilsey ${ }^{3}$ identified an EPR signal labeled NRL-1 with the $V^{2-}$ state of the Ga vacancy by assuming the reverse $A_{1}-T_{2}$ ordering. We therefore disputed that identification and proposed carbon as an alternative. Renewed experimental activity on the EPR signal revealed that the total spin of the center is actually $\frac{3}{2}$, not $\frac{1}{2}$ as originally determined. The calculated ordering of the $A_{1}$ and $T_{2}$ levels made it possible to conclude that NRL-1 originates from the neutral vacancy. This interplay between theory and experiment demonstrated that theory can indeed play a vital role in the difficult task of defect identification.

The results for the antisite defect have been used to give a general description of deep donors in GaP. These donors are similar to the deep donors in Si and can be understood in terms of simple physical models. Comparison of theoretical ionization energies of the antisite defect with experimental values attests to the accuracy and reliability of our calculations and further confirms the identification of the defect.

In the case of carbon, our calculations establish that the center is indeed deep, confirming the general trend that first-row single donors are deep (e.g., $\mathrm{N}$ in $\mathrm{Si}, \mathrm{O}$ in $\mathrm{GaP}$ at a $\mathbf{P}$ site, etc.)

In closing, we wish to make some general remarks about the process of identifying defects. EPR is indeed a very powerful tool. But the extraction of information requires the fitting of data. The total electronic spin of the center is usually a free parameter, but the desire for simplicity has naturally dictated that spin $\frac{1}{2}$ be tried first. In general, if spin $\frac{1}{2}$ fits the data, no higher spin values are tried. The history of NRL-1, however, reveals the dangers inherent in such practice. It is always possible that EPR data which are fit well by assuming spin $\frac{1}{2}$ can be fit equally well by assuming a higher spin. Had this been realized earlier, more possibilities would have been explored for NRL-1. In turn, this realization casts a shadow on other identifications of spin- $\frac{1}{2}$ centers. For example, we now know that the published EPR spectra attributed to the antisite defect can be fit equally well by assuming spin $\frac{3}{2}$. Our calculations, however, make it clear that the $P$ antisite defect can only have spin- $\frac{1}{2}$ and spin-0 deep states. Nevertheless, low-temperature uniaxial-stress data and/or ENDOR data would be desirable to confirm the spin of the center responsible for the EPR signal attributed to the antisite defect.

\section{ACKNOWLEDGMENTS}

This work was supported in part by the U.S. Office of Naval Research under Contract No. N00014-80-C-0679. We would like to thank Patricia Mooney for helpful discussions on the DLTS measurements. 
TABLE III. Multiplet states of the various charge states of the $\mathrm{Ga}$ vacancy in $\mathrm{GaP}$ in the absence of Jahn-Teller distortions. $n_{1}$ is the number of electrons in the $A_{1}$ vacancy level and $n_{2}$ is the number of electrons in the $T_{2}$ vacancy level. When $n_{1}=1$, the vacancy is likely to be in an excited state. The likely lowest-energy multiplet for each charge state is denoted by an asterisk.

\begin{tabular}{|c|c|c|c|c|c|c|}
\hline$n_{1}$ & $n_{2}$ & Charge state & spin 0 & $\operatorname{spin} \frac{1}{2}$ & spin 1 & $\operatorname{spin} \frac{3}{2}$ \\
\hline 2 & 1 & $V^{2+}$ & & ${ }^{2} T_{2}{ }^{*}$ & & \\
\hline 2 & 2 & $V^{+}$ & $\begin{array}{c}{ }^{1} A_{1} \\
{ }^{1} E^{*} \\
{ }^{1} T_{2}\end{array}$ & & ${ }^{3} T_{1}{ }^{*}$ & \\
\hline 2 & 3 & $V^{0}$ & & $\begin{array}{l}{ }^{2} E \\
{ }^{2} T_{1} \\
{ }^{2} T_{2}\end{array}$ & & ${ }^{4} A_{2}{ }^{*}$ \\
\hline 2 & 4 & $V^{-}$ & $\begin{array}{l}{ }^{1} A_{1} \\
{ }^{1} E^{*} \\
{ }^{1} T_{2}\end{array}$ & & ${ }^{3} T_{1}{ }^{*}$ & \\
\hline 2 & 5 & $V^{2-}$ & & ${ }^{2} T_{2}{ }^{*}$ & & \\
\hline 1 & 4 & $V^{0}$ & & $\begin{array}{l}{ }^{2} A_{1} \\
{ }^{2} E \\
{ }^{2} T_{1} \\
{ }^{2} T_{2}\end{array}$ & & ${ }^{4} T_{1}$ \\
\hline 1 & 5 & $V^{-}$ & ${ }^{1} T_{2}$ & & ${ }^{3} T_{2}$ & \\
\hline 1 & 6 & $V^{2-}$ & & ${ }^{2} A_{1}$ & & \\
\hline
\end{tabular}

\section{APPENDIX}

In the main text we gave the results of our singleparticle calculations for the vacancy, the $\mathbf{P}$ antisite defect, and substitutional carbon at $\mathrm{Ga}$ sites. The last two have an $A_{1}$ level in the gap. This level may contain zero, one, or two electrons. None of these states are orbitally degenerate so that no further analysis is necessary. In the case of the vacancy, however, the $T_{2}$ level in the gap may contain any number up to six electrons so that orbital degeneracies are possible. It is then necessary to investigate the various possibilities of occupying the calculated oneelectron levels by forming the many-electron states allowed by Pauli's exclusion principle. The number of many-electron states depends on the number $n_{1}$ of electrons in the $A_{1}$ level inside the valence bands and the number $n_{2}$ of electrons in the $T_{2}$ level in the gap. The procedure is well known and can be found, for example, in
Ref. 37. First, we consider the many-electron states that are possible when $n_{1}=2$. For each value of $n_{2}$, which corresponds to a different charge state, the ground state can be estimated using the Mulliken approximation. ${ }^{37}$ In the first part of Table III we show all the possible manyelectron states for the various charge states of the vacancy when $n_{1}=2$ and identify with asterisks the state or states that are most likely the ground state in the absence of any Jahn-Teller distortions. We note that all spin- $\frac{1}{2}$ states are orbitally degenerate and hence unstable against JahnTeller distortions. In the second part of Table III we list the corresponding states when $n_{1}=1$. These states become relevant if the EPR-active state that gives rise to the NRL-1 signal is not due to a ground state, but rather due to an excited state. This possibility cannot be ruled out if NRL-1 is indeed not seen in the dark in $n$-type samples. However, even for excited states, the only orbitally nondegenerate states have electronic spin $\frac{1}{2}$.
*Present address: Physikalisches-Technische Bundesanstalt (PTB), Braunschweig, Federal Republic of Germany.

†Present address: Corporate Research Science Laboratories, EXXON Research and Engineering Company, Annandale, NJ 08801.

${ }^{1}$ U. Kaufmann, J. Schneider, and A. Räuber, Appl. Phys. Lett. 29, 312 (1976); U. Kaufmann, J. Schneider, R. Wörner, T. A. Kennedy, and N. D. Wilsey, J. Phys. C 14, L951 (1981).

${ }^{2}$ U. Kaufmann and J. Schneider, in Festkörperprobleme (Advances in Solid State Physics), edited by J. Treusch (Vieweg, Braunschweig, 1980), Vol. XX, p. 87.

${ }^{3}$ T. A. Kennedy and N. D. Wilsey, Phys. Rev. Lett. 41, 977 (1978); Phys. Rev. B 23, 6585 (1981).

${ }^{4}$ R. J. Wagner, J. J. Krebs, G. H. Stauss, and A. M. White, Solid
State Commun. $\underline{36}, 15$ (1980).

${ }^{5}$ R. Wörner, U. Kaufmann, and J. Schneider, Appl. Phys. Lett. 40, 141 (1982); E. Weber, H. Ennen, U. Kaufmann, J. Windscheif, J. Schneider, and T. Wosinski, J. Appl. Phys. 53, 6140 (1982).

6J. A. Van Vechten, J. Electrochem. Soc. 122, 423 (1975); J. Electron. Mater. 4, 1159 (1975).

${ }^{7}$ J. Bernholc, N. O. Lipari, and S. T. Pantelides, Phys. Rev. Lett. 41, 895 (1978); Phys. Rev. B 21, 3545 (1980).

${ }^{8}$ G. A. Baraff and M. Schlüter, Phys. Rev. 41, 892 (1978); Phys. Rev. B 19, 4965 (1979).

${ }^{9}$ The method and a number of applications have been reviewed recently by M. Scheffler, in Festkörperprobleme (Advances in Solid State Physics), edited by J. Treusch (Vieweg, Braun- 
schweig, 1982), Vol. 22, p. 115.

${ }^{10} \mathrm{~J}$. Bernholc, S. T. Pantelides, N. O. Lipari, and A. Baldereschi, Solid State Commun. 37, 705 (1982); J. Bernholc, N. O. Lipari, S. T. Pantelides, and M. Scheffler, Phys. Rev. B $\underline{26}$, 5706 (1982).

${ }^{11}$ M. Scheffler, S. T. Pantelides, N. O. Lipari, and J. Bernholc, Phys. Rev. Lett. 47, 413 (1981).

${ }^{12}$ T. A. Kennedy, N. D. Wilsey, J. J. Krebs, and G. H. Stauss, Phys. Rev. Lett. 50, 1281 (1983).

${ }^{13}$ N. D. Wilsey and T. A. Kennedy (private communication).

${ }^{14}$ P. M. Mooney, T. A. Kennedy, and M. B. Small, Physica 116B, 431 (1983).

${ }^{15}$ P. M. Mooney, T. A. Kennedy (unpublished).

${ }^{16}$ The essential elements of the Green's-function formalism are contained in the classic papers by G. F. Koster and J. C. Slater [Phys. Rev. 94, 1392 (1954); ibid. 95, 1165 (1954)], and by J. Callaway [J. Math. Phys. $\underline{5}, 783$ (1964)]. For derivations of the equations in this section, see also F. GarciaMoliner, in Theory of Imperfect Crystalline Solids: Trieste Lectures 1970 (IAEA, Vienna, 1966).

${ }^{17}$ W. Kohn and L. J. Sham, Phys. Rev. 140, A1133 (1965).

${ }^{18}$ L. Hedin and B. I. Lundqvist, J. Phys. C 4, 2064 (1971).

${ }^{19}$ See, e.g., D. R. Hamann, Phys. Rev. Lett. 42, 662 (1979).

20J. R. Chelikowsky and M. L. Cohen, Phys. Rev. B 14, 556 (1976).

${ }^{21}$ G. Gilat and L. J. Raubenheimer, Phys. Rev. 144, 390 (1966).

${ }^{22}$ M. Scheffler, J. P. Vigneron, and S. T. Pantelides (unpublished).

${ }^{23}$ S. T. Pantelides, Bull. Am. Phys. Soc. 26, 255 (1981).

${ }^{24}$ In the second entry in Ref. 3, mass-spectroscopic measurements were reported which find carbon in excess of $2.5 \times 10^{16}$ $\mathrm{cm}^{-3}$. Chemical-analysis studies were later reported [T. A. Kennedy and N. D. Wilsey, Bull. Am. Phys. Soc. 27, 278 (1982)] to reveal very low carbon levels.

${ }^{25}$ N. O. Lipari, J. Bernholc, and S. T. Pantelides, Phys. Rev.
Lett. $\underline{43}, 1354$ (1979).

${ }^{26}$ In the second entry in Ref. 3, it was reported that after irradiation there is an enhancement of optical absorption at energies just below the band gap. Data are not shown for low fluences. It is unlikely that low-fluence irradiation can shrink the gap by as much as $0.6 \mathrm{eV}$ so that electrons at traps near the bottom of the gap can be photoexcited to the conduction band with $1.7-\mathrm{eV}$ photons.

${ }^{27}$ This is a well-known phenomenon in molecular physics. See e.g., P. Avouris, W. M. Gelbert, and M. A. El-Sayed, Chem. Rev. 77, 793 (1977).

${ }^{28}$ H. P. Hjalmarson, P. Vogl, D. J. Wolford, and J. D. Dow, Phys. Rev. Lett. 444, 810 (1980).

${ }^{29}$ G. Ferenczi, L. Dózsa, and M. Somogyi, in Defect Complexes in Semiconductor Structures, edited by J. Griber, F. Beleznay, I. C. Szép, and J. László (Springer, Berlin, 1983), p. 301.

${ }^{30}$ R. L. Aggarwal and A. K. Ramdas, Phys. Rev. 140, A1246 (1965).

${ }^{31}$ K. L. Brower, Phys. Rev. Lett. 44, 1627 (1980).

${ }^{32}$ A. Onton, Phys. Rev. 186, 786 (1969).

${ }^{33}$ See, e.g., P. J. Dean and C. H. Henry, Phys. Rev. 176, 928 (1968); also see G. Baraff, E. O. Kane, and M. Schlüter, Phys. Rev. B $\underline{25}, 548$ (1982).

${ }^{34}$ P. J. Dean, C. J. Frosch, and C. H. Henry, J. Appl. Phys. 39, 5631 (1968).

35J. Ihm, S. G. Louie, and M. L. Cohen, Phys. Rev. B 17, 769 (1978).

${ }^{36}$ Also see the discussion of O. Gunnarsson, H. Hjelmerg, and J. K. Norskov [Phys. Scr. 22, 165 (1980)], and J. Pollmann, in Festkörperprobleme (Advances in Solid State Physics), edited by J. Treusch (Vieweg, Braunschweig, 1980), Vol. 20, p. 117.

${ }^{37}$ See, e.g., M. Lannoo and J. Bourgoin, Point Defects in Semiconductors I (Springer, Berlin, 1981), pp. 141ff; also see M. Lannoo, G. A. Baraff, and M. Schlüter, Phys. Rev. B 24, 943 (1981); 24, 955 (1981). 